\title{
U tilidad de la biopsia de piel en el diagnóstico de enfermedades neurológicas
}

\author{
Pilar de la Sotta $\mathrm{F}^{1}$, William Romero $\mathrm{G}^{1}$, Sergio G onzález B ${ }^{2}$. \\ Skin biopsy for the diagnosis \\ of nervous system diseases
}

Skin biopsy is a powerful diagnostic tool in Dermatology. Its use has been extended to other medical specialties, allowing the diagnosis of several diseases that previously required complex and high risk diagnostic procedures. Skin contains numerous cell types, including blood vessels and peripheral nerves and represents a window to the systemic circulation and nervous system. In this review we discuss the use of skin biopsy to diagnose nervous system diseases in which patients do not exhibit any clinical cutaneous manifestations. We review the usefulness of skin biopsy in cerebral autosomal dominant arteriopathy with subcortical infarcts and leukoencephalopaty (CADASIL), some lysosomal storage diseases, Lafora disease and in peripheral neuropathies (Rev Méd Chile 2008; 136: 1047-55).

(Key words: CADASIL; Diagnostic techniques, surgical, biopsy; Lafora disease; Lysosomal storage)

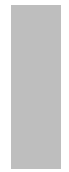

Recibido el 22 de junio, 2007. Aceptado el 5 de septiembre, 2007.

Fuente de financiamiento: ninguna.

Conflictos de interés: no identificados.

Departamentos de ${ }^{1}$ Dermatología y ${ }^{2}$ Anatomía Patológica, Escuela de Medicina, Pontificia Universidad Católica de Chile, Santiago de Chile.
L a biopsia de piel es probablemente la herramienta más importante en Dermatología, no sólo para el diagnóstico, sino para el seguimiento de múltiples dermatosis. Sin embargo, la utilidad de la biopsia de piel se ha extendido a otras áreas de la medicina, especialmente en el campo de las enfermedades neurológicas, donde cambios similares a los observados en el sistema nervioso central pueden verse en la vasculatura o en la inervación cutánea. Así, es posible encontrar

Correspondencia a: Dra. Pilar de la Sotta F. Departamento de Dermatología, Centro Médico San Joaquín, Pontificia Universidad Católica de Chile. Av. Vicuña Mackenna 4686, Macul, Santiago, Chile. Fax: (56 2) 5529974.

E mail: pilardelasotta@yahoo.com alteraciones de la ultraestructura cutánea patognomónicas de enfermedades como la arteriopatía cerebral autosómica dominante con infartos subcorticales y leucoencefalopatía (CADASIL), algunas enfermedades de depósito lisosomal como la mucolipidosis IV y la lipofuscinosis ceroídea neuronal, y otras enfermedades neurodegenerativas como la enfermedad de Lafora. Las enfermedades mencionadas pueden afectar a niños y adultos y, muchas veces, la única forma de hacer el diagnóstico diferencial entre ellas es mediante la biopsia de piel, dado que la clínica no siempre es característica. En este artículo se revisarán estas patologías, con énfasis en la importancia de la biopsia de piel para su diagnóstico. En forma adicional, se indica sitio de biopsia y técnica específica para el diagnóstico de cada patología. 
ARTERIOPATÍA CEREBRAL AUTOSÓMICA DOMINANTE CON INFARTOS SUBCORTICALES Y LEUCOENCEFALOPATÍA (CADASIL)

La arteriopatía cerebral autosómica dominante con infartos subcorticales y leucoencefalopatía (CADASIL, Cerebral Autosomal Dominant Arteriopathy with Subcortical Infarcts and Leukoencephalopathy) es una enfermedad cerebrovascular hereditaria caracterizada por accidentes vasculares isquémicos recurrentes, que se manifiesta en la adultez y progresa a demencia ${ }^{1,2}$. Más de 400 familias afectadas y algunos casos esporádicos, han sido descritos ${ }^{3}$. Actualmente se piensa que podría ser responsable de muchos de los casos previamente diagnosticados como demencia multinfarto idiopática ${ }^{4}$.

a) Etiología: Se produce por mutación del gen Notch3, localizado en el cromosoma 19q125. Notch 3 es un receptor transmembrana y pertenece a la familia de receptores altamente conservados Notch/LN-12 que participan en la determinación del destino celular durante la embriogénesis ${ }^{5,6}$; sin embargo, en adultos sólo se expresa en células de músculo liso vascular y su función es desconocida ${ }^{7,8}$. Notch 3 contiene 34 repeticiones en tándem tipo EGF. Las mutaciones patológicas se localizan dentro de los exones que codifican las secuencias repetidas, y resultan en la ganancia o pérdida de un residuo de cisteína en el domino extracelular 2,3,5,9.

b) Manifestaciones clínicas: Se caracteriza por presentar problemas de aprendizaje durante la infancia ${ }^{10}$, migraña y depresión en la juventud, e infartos subcorticales con déficit neurológico progresivo en la adultez ${ }^{11}$. Las manifestaciones clínicas de CADASIL generalmente comienzan con cefalea tipo migraña, que progresa con ataques isquémicos transitorios hacia una demencia multinfarto durante la sexta o séptima década de la vida $^{11-13}$. En una revisión de 102 pacientes con CADASIL $^{14}$, la forma de presentación más frecuente fue como episodios isquémicos recurrentes, tipo accidentes isquémicos transitorios, en el $71 \%$ de los casos. Otras manifestaciones son: alteración de la marcha (90\%), incontinencia urinaria $(86 \%)$, parálisis pseudobulbar (52\%), déficit cognitivo (48\%), migraña (38\%), de las cuales $87 \%$ tienen aura, alteraciones psiquiátricas (30\%), demencia (28\%), desorden de adaptación (24\%) y convulsiones $(10 \%)^{14,15}$. Los pacientes fallecen, en promedio, 20 años después de iniciados los síntomas.

c) Diagnóstico: La resonancia nuclear magnética de cerebro muestra grados variables de hiperintensidad dentro de la sustancia blanca subcortical y periventricular, con infartos lacunares en tálamo, ganglios basales y troncoencéfalo ${ }^{16-18}$, sin embargo, estos cambios no son específicos de CADASIL y pueden estar ausentes hasta etapas avanzadas de la enfermedad ${ }^{11,19}$. Existen estudios genéticos para mutaciones del gen Notch 3, sin embargo, son caros y no fácilmente disponibles.

El diagnóstico específico de CADASIL se basaba en los hallazgos de la biopsia de cerebro y, en muchos casos, se hacía en forma retrospectiva durante la autopsia.

En la biopsia de cerebro, se observa daño difuso de la sustancia blanca, sin cambios en la corteza, con fibrosis, degeneración del músculo liso vascular con cambios granulares ácido periódico-Schiff (PAS) positivos en la túnica media, y obliteración de las arterias perforantes pequeñas y arterias meníngeas ${ }^{11,20}$. El estudio con microscopía electrónica $(\mathrm{ME})$ de la vasculatura cerebral revela degeneración y destrucción de las células musculares lisas de las arterias pequeñas, observándose característicamente depósitos de material granular osmiofílico (MGO) alrededor de las células de músculo liso ${ }^{21,22}$. Se ha postulado que las mutaciones en CADASIL podrían alterar el reciclaje del ectodominio de Notch3 desde la superficie celular lo que explicaría la acumulación del $\mathrm{MGO}^{7}$.

Los depósitos de MGO son patognomónicos de CADASIL y fueron, posteriormente, encontrados en arterias de otros órganos, como corazón, riñón, intestino delgado y grueso, hígado, músculo esquelético y piel ${ }^{23,24}$. Este descubrimiento demostró que CADASIL es una arteriopatía sistémica y, por otro lado, facilitó el diagnóstico, ya que evidenció que los cambios observados en el cerebro se correlacionan con los de la piel.

d) Biopsia de piel: La biopsia de piel es un método fácil y efectivo, para detectar el MGO en pacientes con sospecha de CADASIL, por lo que se ha 
convertido en el método diagnóstico de elección ${ }^{23-}$ 27. El MGO a la ME se observa en la membrana basal de las células musculares lisas vasculares de los capilares dérmicos (Figura 1) 21,22,28.

Se utiliza biopsia de piel tipo sacabocado ("punch") de 2 a $3 \mathrm{~mm}$ de diámetro de cualquier zona del cuerpo, que debe ser inmediatamente fijada con glutaraldehído al $2 \%$ para el estudio con $\mathrm{ME}^{4}$.

Múltiples estudios han determinado que la presencia de MGO en las arterias dérmicas alcanzaría una especificidad de 100\% para el diagnóstico de CADASIL En uno de ellos, todas las 14 muestras tomadas de individuos afectados, incluyendo 2 portadores asintomáticos de mutaciones en Notch3, mostraron los depósitos vasculares de $\mathrm{MGO}^{29}$. Sin embargo, existe la posibilidad de falsos negativos debido a la naturaleza focal del MGO, lo que se evita realizando múltiples cortes profundos 30,31 .

Existe una estrecha correlación entre los cambios observados en los capilares dérmicos y los encontrados en las arterias cerebrales, lo que sugiere que la biopsia de piel también podría ser útil en la evaluación de la actividad de la enferme$\operatorname{dad}^{26}$. Por otro lado, los cambios cutáneos preceden a los cambios detectables en neuroimágenes y están presentes en portadores asintomáticos, por lo que la biopsia de piel también es útil en el estudio de familiares ${ }^{30,32}$.
En la mayoría de los casos, es posible sospechar el diagnóstico de CADASIL con los antecedentes clínicos y las neuroimágenes, especialmente si son compatibles con eventos isquémicos en pacientes sin factores de riesgo. En estos casos el examen diagnóstico de elección es la biopsia de piel con estudio de ME. Un mayor grado de sospecha clínica, sumado a la disponibilidad de la biopsia de piel, probablemente permitirá diagnosticar muchos casos previamente subdiagnosticados.

\section{ENFERMEDADES DE DEPÓSITO LISOSOMAL}

Las enfermedades de depósito lisosomal (EDL) son un grupo heterogéneo de patologías producidas por defectos en la función de los lisosomas, organelos responsables de la digestión y reciclaje de diversas macromoléculas ${ }^{33}$. La mayoría de las EDL son causadas por defectos hereditarios, sin embargo, también existen formas adquiridas. Hasta la fecha se han descrito 51 entidades de causa genética $^{33}$. La incidencia combinada de las EDL se ha estimado entre 1 - 4 por 100.000 habitantes ${ }^{34}$, con variaciones regionales, llegando a 1 en 5.0008.000 en ciertas zonas de Australia, Europa y Estados Unidos de Norteamérica ${ }^{35}$. Dependiendo del sitio de la mutación y/o del gen defectuoso, las EDL pueden manifestarse en forma infantil o adulta $^{36}$.

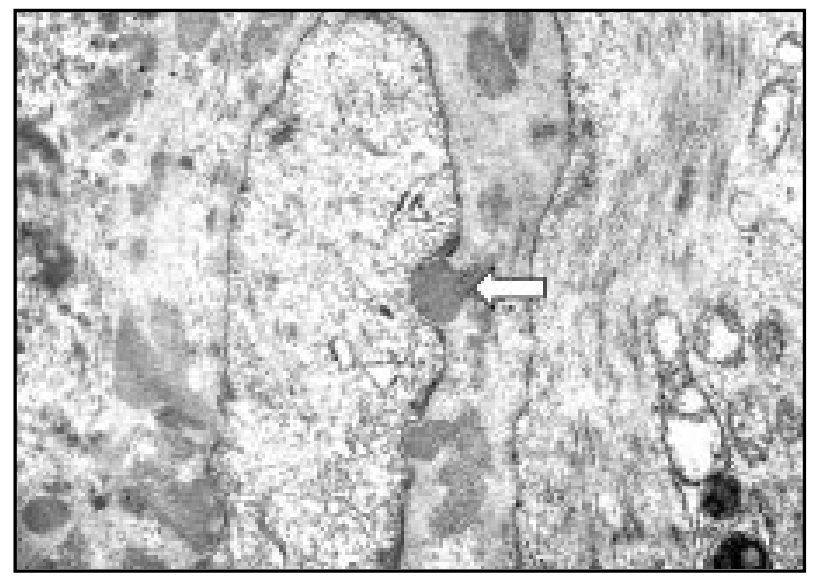

Figura 1. Arteriopatía cerebral autosómica dominante con infartos subcorticales y leucoencefalopatía (CADASIL). Paciente de sexo masculino de 59 años con deterioro orgánico cerebral progresivo. La microscopía electrónica de la biopsia de piel muestra material granular osmiofílicio (flecha) en las células musculares lisas vasculares de los capilares dérmicos (ME, $80.000 \mathrm{x}$ ). 
a) Etiología: Las EDL pueden ser producidas por deficiencias en la actividad de las enzimas lisosomales (hidrolasas ácidas), defectos en la destinación de las enzimas al lisosoma, defectos de transporte en la membrana lisosomal, o deficiencias en proteínas activadoras (saposinas).

b) Manifestaciones clínicas: Las manifestaciones de las EDL dependen del tipo de mutación y de su interacción con factores aún desconocidos. La clínica no es específica para una EDL determinada, pero dependiendo del tipo y naturaleza de la mutación, la enfermedad puede presentarse en forma infantil o de la adultez. Las alteraciones más frecuentes son desórdenes neurológicos, hepatoesplenomegalia, opacidad corneal y anomalías esqueléticas, en forma aislada o combinada ${ }^{38}$. Estas manifestaciones también pueden ser producidas por enfermedades no lisosomales, por lo que en general las EDL representan un enorme reto para el clínico.

c) Diagnóstico: En la mayoría de las EDL, el diagnóstico requiere demostrar la deficiencia de una única enzima en el plasma, leucocitos o cultivo de fibroblastos, o identificar la anomalía genética específica ${ }^{33,37}$. Sin embargo, el dilema para el clínico es sospechar la enfermedad, dado que los signos son inespecíficos. Por otro lado, el costo de estudiar todas las posibles enzimas y genes es extremadamente alto ${ }^{37}$. En este contexto, es esencial disponer de un examen que permita orientar hacia una enfermedad específica. Se ha demostrado que existen patrones ultraestructurales en los lisosomas enfermos relativamente específicos $\mathrm{y}$, algunos, patognomónicos de una determinada EDL, y por ello el estudio histopatológico se ha convertido en una poderosa herramienta en el estudio de estas patologías. Para el diagnóstico de EDL se ha utilizado la ME de biopsias de conjuntiva ${ }^{38}$, recto ${ }^{39}$ y piel ${ }^{40}$. La biopsia de piel es la más simple y mejor tolerada, y además permite descartar enfermedades con manifestaciones similares como CADASIL y otras patologías neurodegenerativas ${ }^{22}$.

d) Biopsia de piel: La ME de piel es la herramienta diagnóstica de tamizaje más eficiente y costoefectiva para las EDL, con una sensibilidad mayor al $90 \% 33,37$.
Para el estudio de las EDL se han utilizado biopsias tipo sacabocado de piel de la región axilar $^{41}$ y del antebrazo ${ }^{37}$. La región axilar podría ser superior para el estudio de enfermedades metabólicas debido a que se pueden encontrar múltiples tipos celulares en una sola muestra de piel, especialmente epitelio ductal y glandular de glándulas sudoríparas, células endoteliales, músculo liso, fibroblastos, pericitos y células de Schwann ${ }^{41}$.

El diagnóstico morfológico de las EDL por ME depende de la observación de numerosos lisosomas secundarios aumentados de tamaño en las células afectadas y, aunque el defecto genético esté presente en todas las células del individuo, sólo algunas células presentan alteraciones.

La acumulación de material de depósito en un tipo celular específico es determinada por la estructura química y la cantidad de sustrato que es reciclado por esas células ${ }^{42}$. Mediante la identificación de los tipos celulares con lisosomas enfermos y las características ultraestructurales del material acumulado, es posible determinar si el defecto interfiere con el catabolismo de glicolípidos, mucopolisacáridos, glicoproteínas o lípidos. Sin embargo, es raro que la morfología del material almacenado sea patognomónica ${ }^{34}$.

En general existen tres apariencias ultraestructurales principales del material almacenado ${ }^{37}$.

a) Estructuras membranosas laminares, se observan por acumulación de glicolípidos, como gangliosidos y esfingomielina, y pueden tener apariencia de "huellas dactilares" o "cuerpos de cebra" (Figura 2).

b) Lisosomas vacíos o material fibrilar fino, se observan por depósitos de oligosacáridos, ácido siálico libre o mucopolisacáridos.

c) Combinación de estructuras membranosas laminares con material fibrilar en el mismo lisosoma, que se produce por inhibición secundaria de enzimas por un primer sustrato ya acumulado, con depósito simultáneo de oligosacáridos y glicolípidos.

A continuación se mencionan en forma breve las características ultraestructurales, de algunas EDL específicas:

Mucopolisacaridosis: se caracterizan por actividad deficiente de enzimas que degradan glicosaminoglicanos y se observa en forma simultánea mate- 


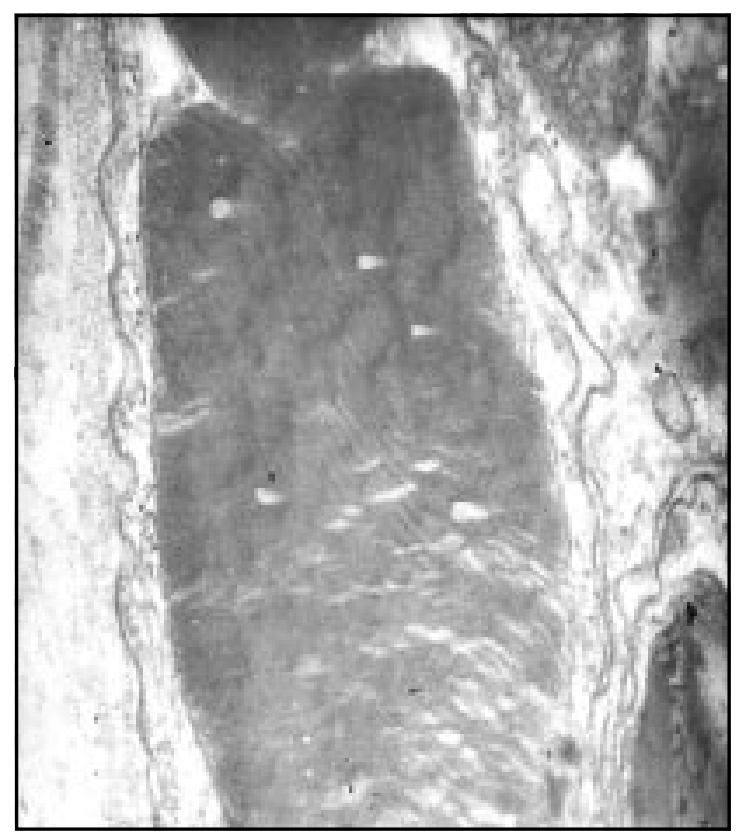

Figura 2. Enfermedad de Fabry o angioqueratoma corporal difuso. Paciente de sexo femenino de 51 años que se presentó con múltiples angioqueratomas. Se observan estructuras membranosas laminares por depósitos de glicoesfingolípidos (ME, 120.000 x).

rial fibrilar fino y estructuras membranosas laminares dentro de un mismo lisosoma ${ }^{43}$.

Mucolipidosis: se caracterizan por deficiencias en el metabolismo de glicolípidos, destacando la mucolipidosis $\mathrm{IV}$, donde se observan estructuras membranosas laminares en los lisosomas de múltiples tejidos, excepto en neuronas, y donde la ME es esencial para el diagnóstico ${ }^{44}$.

Enfermedades con alteración del metabolismo de esfingolípidos: en este grupo destacan las enfermedades de Fabry, de Niemann-Pick, de Krabbe y de Gaucher. La enfermedad de Fabry se caracteriza por depósitos de glicoesfingolípidos y se observan estructuras membranosas laminares en múltiples células (Figura 2), siendo distintiva la presencia de múltiples capas de lámina basal alrededor de los vasos sanguíneos ${ }^{45,46}$. En la enfermedad de Niemann-Pick se acumula esfingomielina que aparece como estructuras membranosas laminares más gruesas ${ }^{47}$. En la enfermedad de
Krabbe se deposita galactosilesfingosina y se observan estructuras tubulares en las células de Schwann e inclusiones tipo hendidura en las glándulas sudoríparas ecrinas ${ }^{48,49}$. La enfermedad de Gaucher se caracteriza por depósitos de glucosilceramida en células de estirpe monocito/macrófago en bazo, médula ósea, hígado, ganglios, pulmones, corazón y cerebro, pero no en piel ${ }^{50}$.

Enfermedades con alteración del catabolismo de glicoproteínas: en este grupo destacan la fucosidosis, las manosidosis y la enfermedad Pompe. La fucosidosis se caracteriza porque la enzima defectuosa cataboliza glicolípidos y oligosacáridos, observándose glicolípidos (estructuras membranosas laminares) en las células de Schwann y oligosacáridos (material fibrilar fino) en las células endoteliales adyacentes ${ }^{45}$. En las manosidosis se acumulan oligosacáridos, observándose lisosomas vacíos o con material fibrilar. En la enfermedad de Pompe se acumula glicógeno observándose partículas electróndensas de aproximadamente $30 \mathrm{~nm}$ de diámetro.

Lipofuscinosis ceroídea neuronal (LCN): se conocen 9 variedades de LCN, cada una con depósitos de material morfológicamente diferentes ${ }^{51-53}$. Algunas de las formas observadas son: depósitos granulares osmiofílicos, perfiles curvilíneos, "huellas dactilares" y complejos rectilíneos (Figura 3). Algunas de las variantes de LCN tienen material de depósito morfológicamente uniforme, mientras que otras tienen mezclas de estructuras. Actualmente la $\mathrm{ME}$ es el único método disponible para el diagnóstico de LCN.

Finalmente, el estudio con ME de biopsias cutáneas representa el test diagnóstico inicial más efectivo en la evaluación de pacientes con sospecha de EDL, y permite orientar el estudio enzimático o genético específico.

\section{ENFERMEDAD DE LAFORA}

La enfermedad de Lafora (EL) es una enfermedad genética autosómica recesiva perteneciente al grupo de las epilepsias mioclónicas progresivas. Se caracteriza por degeneración del sistema nervioso central con aparición de cuerpos intracelulares PAS positivos, conocidos como cuerpos de Lafora 


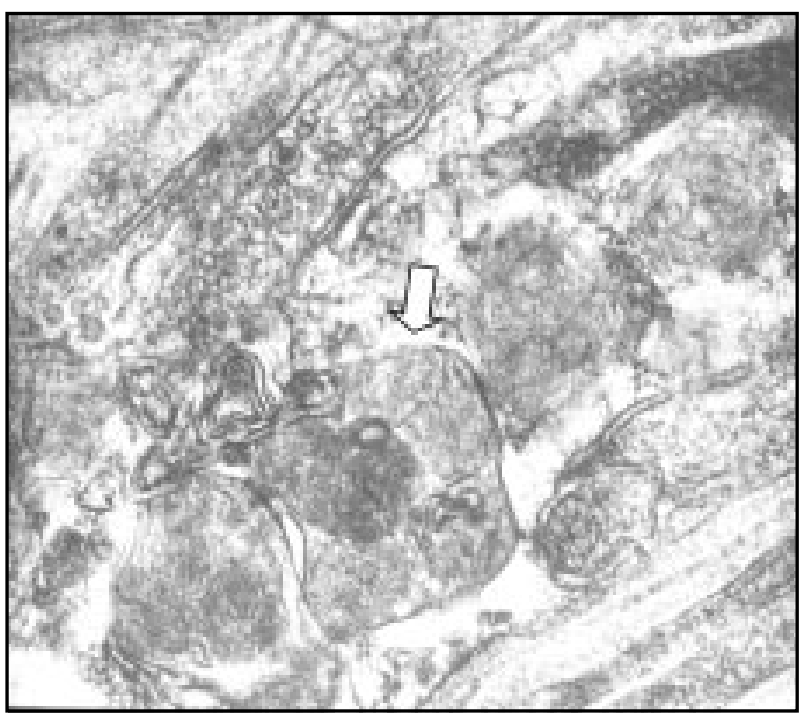

Figura 3. Lipofuscinosis ceroídea neuronal. Paciente de sexo masculino de 4 años de edad con deterioro psicomotor progresivo. Se observan depósitos laminares y granulares al interior de los lisosomas (flecha) (ME, $120.000 \mathrm{x})$.

(CL), en múltiples tejidos como cerebro, piel e hígado (Figura 4$)^{54}$. La prevalencia de la enfermedad es desconocida, sin embargo, la mayoría de los casos se han descrito en Europa y provienen de matrimonios consanguíneos ${ }^{55}$.

a) Etiología. La EL es producida por mutación del gen EPM2A, localizado en el cromosoma 6q23-25, en el $75 \%-85 \%$ de los casos $^{56}$. Este gen codifica una proteína de 331 aminoácidos con función tirosina fosfatasa, conocida como laforina, que se localiza en la membrana plasmática, el retículo endoplásmico y los poliribosomas ${ }^{57}$. Esta proteína además contiene una molécula de unión a carbohidratos con la cual parece unirse a glicógeno ${ }^{56}$. No se conoce la función biológica de laforina, pero en la EL la proteína pierde su actividad.

Los CL están compuestos principalmente de polímeros de glucosa (poliglucosanos) y se acumulan principalmente en las células piramidales largas de la tercera y cuarta lámina de la corteza cerebral y en el pericarion.

b) Clínica. La EL comienza a manifestarse en la segunda década de la vida, en promedio a los 14 años $^{55}$, aunque puede ser de inicio tardío. Con- vulsiones tónico-clónicas generalizadas son en general la primera manifestación, y menos frecuentemente mioclonías o demencia. Otras manifestaciones son convulsiones occipitales con aura de luces de colores, mioclonías, signos cerebelosos, hiperreflexia y alteraciones visuales. Todos los casos desarrollan demencia rápidamente progresiva con disfunción cognitiva global en 2 a 6 años. La muerte ocurre 2 a 10 años después de iniciados los síntomas, en promedio a los 20 años de edad ${ }^{55}$.

c) Diagnóstico. Las neuroimágenes pueden ser normales o se observa atrofia cerebral difusa que aumenta con la progresión de la enfermedad ${ }^{55}$. El examen diagnóstico de elección es la biopsia de piel de la región axilar que permite identificar los CL

d) Biopsia de piel. La biopsia de piel tiene una sensibilidad de 80\%-100\% para el diagnóstico de $\mathrm{EL}^{55}$. El sitio de elección para la biopsia es la región axilar ya que permite identificar los CL en los ductos de las glándulas sudoríparas ecrinas y apocrinas y en las células mioepiteliales del acino secretor de las glándulas sudoríparas apocrinas (Figura 4) ${ }^{58}$. 


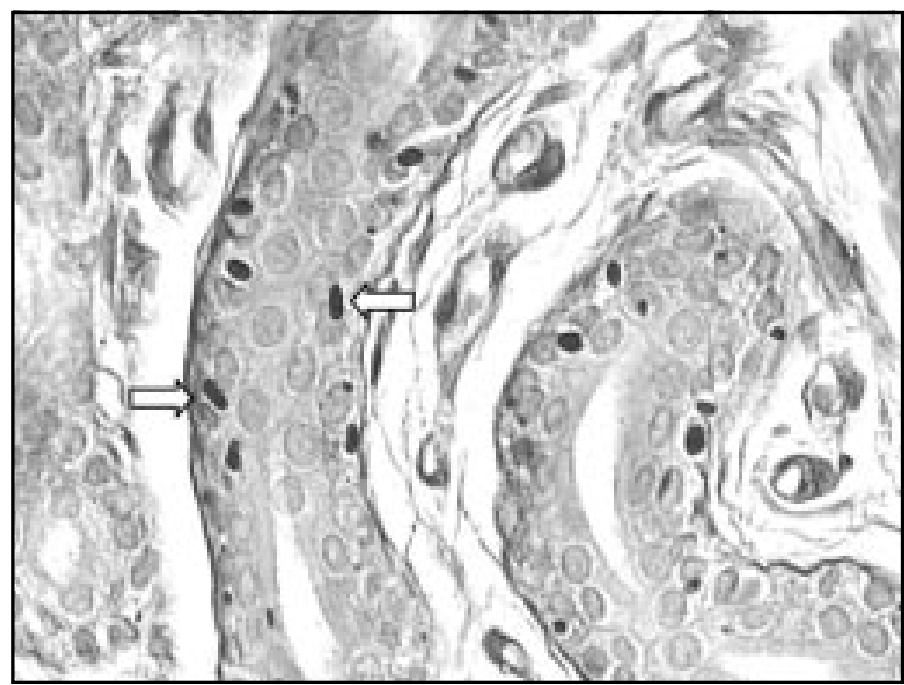

Figura 4. Enfermedad de Lafora. Se observan los cuerpos de Lafora (flechas) como cuerpos intracelulares PAS (+) en los ductos de las glándulas sudoríparas ecrinas (PAS, 200 x).

\section{NEUROPATÍAS PERIFÉRICAS}

La biopsia de piel se ha utilizado para el diagnóstico de diferentes neuropatías periféricas, incluyendo neuropatías autonómicas y desmielinizantes.

En el año 2004 la Federación Europea de Sociedades Neurológicas desarrolló guías para el uso de la biopsia de piel en el diagnóstico de neuropatías periféricas ${ }^{59}$. Se recomendó realizar biopsias tipo sacabocado de $3 \mathrm{~mm}$ de diámetro en la zona distal de la pierna y cuantificar la densidad linear de las fibras nerviosas intraepidérmicas nomielinizadas (FNIE) en muestras marcadas con anticuerpos anti-PGP 9.5, un marcador neuronal citoplasmático. Así, la densidad de FNIE se correlaciona estrechamente con el umbral al calor y dolor, y parece ser más sensible que los estudios de conducción y que la biopsia del nervio sural, para el diagnóstico de neuropatía sensorial de fibras pequeñas ${ }^{59}$. Por otro lado, el estudio en el tiempo de la densidad de FNIE y las tasas de regeneración se correlacionan con la progresión de las neuropatías ${ }^{59}$.

\section{CONCLUSIONES}

La utilidad de la biopsia de piel ha sobrepasado el ámbito exclusivo de la dermatología y, actualmente, es un método diagnóstico para múltiples patologías neurológicas.

Para la mayonía de las patologías discutidas en esta revisión se requiere el uso de microscopía electrónica de transmisión, que requiere de una técnica, instrumental y personal altamente especializados. Sin embargo, el costo actual es relativamente bajo y es asequible a todo tipo de pacientes y formas de financiamiento existentes en nuestro país. Para realizar este examen y obtener muestras de óptima calidad y la mejor información posible de las biopsias, es necesania una comunicación previa entre el equipo tratante y el laboratorio de Anatomía Patológica para acordar el lugar de toma de muestra y la mejor forma de fijación para su envío al laboratorio.

En este artículo intentamos sistematizar el uso de la biopsia de piel en enfermedades sistémicas sin manifestaciones cutáneas, y así facilitar la interacción de los distintos especialistas ante la sospecha de alguna de estas patologías. 


\section{REFERENCIAS}

1. SOURANDER P, WALINDER J. Hereditary multi-infanct dementia. Morphological and clinical studies of a new disease. Acta Neuropathol (Berl) 1977; 39: 247-54.

2. Tournier-Lasserve E, Joutel A, Melki J, Weissenbach J, Lathrop GM, Chabriat H et al. Cerebral autosomal dominant arteriopathy with subcortical infarcts and leukoencephalopathy maps to chromosome 19q12. Nat Genet 1993; 3: 256-9.

3. Joutel A, Dodick DD, Parisi JE, CeciLon M, TournierLASSERVE E, BOUSSER MG. De novo mutation in the Notch3 gene causing CADASIL Ann Neurol 2000; 47: 388-91.

4. Rumbaugh Ja, LaDuca JR, Shan Y, Milier CA. CadaSIL: the dermatologic diagnosis of a neurologic disease. Cerebral autosomal-dominant arteriopathy with subcortical infarcts and leukoencephalopathy. J Am Acad Dermatol 2000; 43: 1128-30.

5. Joutel A, Corpechot C, Ducros A, Vahedi K, Chabriat H, Mouton P ET AL. Notch3 mutations in CADASIL, a hereditary adult onset condition causing stroke and dementia. Nature 1996; 383: 707-10.

6. ARTAVANis-TSaKonas S, RAND MD, LaKe RJ. Notch signaling: cell fate control and signal integration in development. Science 1999; 284: 770-6.

7. Joutel A, Andreux F, Gaulis S, Domenga V, Cecilon M, Battail N et aL The ectodomain of the Notch3 receptor accumulates within the cerebrovasculature of CADASIL patients. J Clin Invest 2000; 105: 597-605.

8. Vima N, Walker L, LndSell CE, Gasson J, Iruela-Arispe ML, Weinmaster G. Vascular expression of Notch pathway receptors and ligands is restricted to arterial vessels. Mech Dev 2001; 108: 161-4.

9. Kalimo H, Vittanen M, Amberla K, Juvonen V, Marttila $R$, Poyhonen M et aL. CADASIL: hereditary disease of arteries causing brain infarcts and dementia. Neuropathol Appl Neurobiol 1999; 25: 257-65.

10. Desmond DW, Moroney JT, Lynch T, Chan S, Chin SS, Shungu DC et aL CADASIL in a North American family: clinical, pathologic, and radiologic findings. Neurology 1998; 51: 844-9.

11. Chabriat H, Vahedi $K$, Iba-Zizen MT, Joutel A, Nibio A, Nagy TG ET AL. Clinical spectrum of CADASIL: a study of 7 families. Cerebral autosomal dominant arteriopathy with subcortical infarcts and leukoencephalopathy. Lancet 1995; 346: 934-9.

12. Davous P. The natural history of CADASIL Stroke 1999; 30: 22-47.

13. Desmond DW, Moroney JT, Lynch T, Chan S, Chin SS, MoHR JP. The natural history of CADASIL: a pooled analysis of previously published cases. Stroke 1999; 30: 1230-3.

14. Dichgans M, Mayer M, Uttner I, Bruning R, MulerHOCKER J, RungGER G ET AL. The phenotypic spectrum of CADASIL: clinical findings in 102 cases. Ann Neurol 1998; 44: 731-9.
15. Kantakis J, Thobois S, Ciaudy A, Broussoue E. CADASIL (cerebral autosomal dominant arteriopathy with subcortical infarcts and leukoencephalopathy): a neurovascular disease diagnosed by ultrastructural examination of the skin. J Cutan Pathol 2002; 29: 498-501.

16. Chabriat H, Levy C, Taima H, Iba-Zizen MT, Vahedi K, Joutel A ET AL. Patterns of MRI lesions in CADASIL. Neurology 1998; 51: 452-7.

17. Chabriat $H$, Mrissa R, Levy $C$, Vahedi $K$, Taima $H$, IbaZizen MT ET AL. Brain stem MRI signal abnormalities in CADASIL. Stroke 1999; 30: 457-9.

18. Yousry TA, Seelos K, Mayer M, Bruning R, Uttner I, Dichgans M ET AL. Characteristic MR lesion pattern and correlation of T1 and T2 lesion volume with neurologic and neuropsychological findings in cerebral autosomal dominant arteriopathy with subcortical infarcts and leukoencephalopathy (CADASIL). AJNR Am J Neuroradiol 1999; 20: 91-100.

19. Chabriat $H$, Taila H, Iba-Zizen MT, Levy $C$, Vahedi $K$, ABANOU A ET AL MRI features of cerebral autosomal dominant arteriopathy with subcortical infarcts and leukoencephalopathy. Neurology 1996; 46 (Suppl): A212.

20. Walsh JS, Perniciaro C, Meschia JF. CADASIL (cerebral autosomal dominant arteriopathy with subcortical infarcts and leukoencephalopathy): diagnostic skin biopsy changes determined by electron microscopy. J Am Acad Dermatol 2000; 43: 1125-7.

21. Ruchoux MM, Maurage CA. CADASIL: Cerebral autosomal dominant arteriopathy with subcortical infarcts and leukoencephalopathy. J Neuropathol Exp Neurol 1997; 56: 947-64.

22. Ruchoux MM, Maurage CA. Endothelial changes in muscle and skin biopsies in patients with CADASIL. Neuropathol Appl Neurobiol 1998; 24: 60-5.

23. Ruchoux MM, CHABRiat H, Bousser MG, BaudRimont M, TouRNIER-LASSERVE E. Presence of ultrastructural arterial lesions in muscle and skin vessels of patients with CADASIL. Stroke 1994; 25: 2291-2.

24. Ruchoux MM, Guerrouadou D, Vandenhaute B, Pruvo JP, VERMERSCH P, LeYs D. Systemic vascular smooth muscle cell impairment in cerebral autosomal dominant arteriopathy with subcortical infarcts and leukoencephalopathy. Acta Neuropathol 1995; 89: 500-12.

25. Schroder JM, Seluhaus B, Jorg J. Identification of the characteristic vascular changes in a sural nerve biopsy of a case with cerebral autosomal dominant arteriopathy with subcortical infarcts and leukoencephalopathy (CADASIL). Acta Neuropathol (Berl) 1995; 89: 116-21.

26. Bruln P, Godfraind C, Leteurtre E, Ruchoux MM. Morphometric analysis of ultrastructural vascular changes in CADASIL: analysis of 50 skin biopsy specimens and pathogenic implications. Acta Neuropathol (Berl) 2002; 104: 241-8.

27. Ishiko A, Shimizu A, Nagata E, Ohta K, Tanaka M. Cerebral autosomal dominant arteriopathy with subcortical infarcts and leukoencephaloapthy (CADA- 
SIL): a hereditary cerebrovascular disease, which can be diagnosed by skin biopsy electron microscopy. Am J Dermatopathol 2005; 27: 131-4.

28. Baudrimont M, Dubas F, Joutel A, Tournier-Lasserve E, Bousser MG. Autosomal dominant leukoencephalopathy and subcortical ischemic stroke: a clinicopathologic study. Stroke 1993; 24: 122-5.

29. Mayer M, Straube A, Bruening R, Uttner I, Pongratz D, Gasser T ET AL. Muscle and skin biopsies are a sensitive diagnostic tool in the diagnosis of CADASIL. J Neurol 1999; 246: 526-32.

30. Rubio A, Rffkin D, Powers JM, Patel U, Stewart J, Faust P et AL Phenotypic variability of CADASIL and novel morphologic findings. Acta Neuropathol 1997; 94: 247-54.

31. Schultz A, Santoianni R, Hewan-Lowe K. Vasculopathic changes of CADASIL can be focal in skin biopsies. Ultrastruct Pathol 1999; 23: 241-7.

32. Ebke M, Dichgens M, Bergmann M, Voelter HU, Rieger P, GASSER T ET AL. CADASIL skin biopsy allows diagnosis in early stages. Acta Neurol Scand 1997; 95: 351-7.

33. Alroy J, Ucci AA, Pfannl R. Why skin biopsy is useful for the diagnosis of lysosomal storage diseases. Current Medical Literature: Lysosomal Storage Disease 2006; 5: 70-6.

34. Reuser AJJ, Kroos MA, Visser WJ, WiLemsen R. Lysosomal storage diseases: cellular pathology, clinical and genetic heterogeneity, therapy. Ann Biol Chem 1994; 52: 721-8.

35. Wenger DA, Coppola S, Lu S-L. Insight into the diagnosis and treatment of lysosomal storage diseases. Arch Neurol 2003; 60: 322-8.

36. NeUFELD EF. Natural history and inherited disorders of lysosomal enzyme, b-hexosaminidase. J Biol Chem 1989; 264: 10927-30.

37. Alroy J, Ucci AA. Skin biopsy: a useful tool in the diagnosis of lysosomal storage diseases. Ultrastruct Pathol 2006; 30: 489-503.

38. Merin S, Lunn N, Berman ER, Yatziv S. Mucolipidosis, IV: ocular, systemic and ultrastructural findings. Invest Ophthalmol 1975; 14: 437-48.

39. Ushiyama M, Ikeda S, Nakayama J, Yanagisawa N, Hanyu N, KatsuYama T. Type III (chronic) GM1-gangliosidosis. Histochemical and ultrastructural studies of rectal biopsy. J Neurol Sci 1985; 71: 209-23.

40. BELCHER RW. Ultrastructure of the skin in the genetic mucopolysccharidoses. Arch Pathol 1972; 94: 511-8.

41. Abramovich CM, Prayson RA, McMahon JT, Cohen BH. Ultrastructural examination of the axillary skin biopsy in the diagnosis of metabolic diseases. Hum Pathol 2001; 32: 649-55.

42. Castagnaro M, Alroy J, Ucci AA, Glew RH. Lectin histochemistry and ultrastructure of feline kidneys from six different storage diseases. Virchows Arch B Cell Pathol 1987; 54: 16-26.

43. Avila JL, Convit J. Inhibition of leukocytes lysosomal enzymes by glycosaminoglycans in vitro. Biochem J 1975; 152: 57-64.
44. Folkerth RD, Alroy J, LOMAKina I, SkUtelsky E, Raghavan SS, Kolodny EH. Mucolipidosis IV: morphology and histochemistry of an autopsy case. J Neuropathol Exp Neurol 1995; 54: 154-64.

45. Prasad A, Kaye EM, Alroy J. Electron microscopic examination of skin biopsy as a cost-effective tool in the diagnosis of lysosomal storage disease. J Child Neurol 1996; 11: 301-8.

46. Dvorak AM, Cabie WJ, Osage JE, Kolodny EH. Diagnostic electron microscopy. II. Fabry's disease: use of biopsies from uninvolved skin. Acute and chronic changes involving the microvasculature and small unmyelinated nerves. Pathol Annu 1981; 16 Pt 1: 139-58.

47. Boustany R-MN, Kaye E, Alroy J. Ultrastructural findings in skin from patients with Niemann-Pick disease, type C. Pediatr Neurol 1990: 6: 177-83.

48. ELIEDER M. Eccrine gland involvement in Krabbe's disease. Virchows Arch A Pathol Anat Histopathol 1992; 421: 351-4

49. Ceuterick C, Martin J-J. Krabbe globoid cell leukodystrophy, electron microscopy shows characteristic inclusions in eccrine sweat gland. Path Res Pract 1993: 189: 384-6.

50. Beutler E, Grabowski GA. Gaucher disease. In: Scriver R, Beaudet AL, Sly WS et al, eds. The Metabolic and Molecular Base of Inherited Disease, ed 8. New York: McGraw-Hill; 2001: 3635-68.

51. Schulz A, Dhar S, Rylova S, Dbaibo G, Alroy J, Hagel C ET AL. Impaired cell adhesion and apoptosis in a novel CLN9 Batten disease variant. Ann Neurol 2004; 56: 342-50.

52. Haltia M. The neuronal ceroid-lipofuscinoses. J Neuropathol Exp Neurol 2003: 62: 1-13.

53. Wisniewsi KE, Zhong N. PhiLppart M. Pheno/genotypic correlations of neuronal ceroid lipofuscinoses. Neurology 2001; 57: 576-81.

54. Zupanc ML, Legros B. Progressive myoclonic epilepsy. Cerebellum 2004; 3: 156-71.

55. Acharya JN, Satischandra P, Asha T, Shankar SK. Lafora's disease in south India: a clinical, electrophysiologic, and pathologic study. Epilepsia 1993; 34: 476-87.

56. Chan EM, Bulman DE, Paterson AD, Turnbull J, ANDERMANN E, ANDERMANN F ET AL. Genetic mapping of a new Lafora progressive myoclonus epilepsy locus (EPM2B) on 6p22. J Med Genet 2003; 40: 671-5.

57. Minassian BA, Andrade DM, Ianzano L, Young EJ, CHAN E, ACKERLEY CA ET AL. Laforin is a cell membrane and endoplasmic reticulum-associated protein tyrosine phosphatase. Ann Neurol 2001; 49: 271-5.

58. IANNACCONE S, ZuCCONI M, QuATtrini A, NEMNI R, Comola M, Taccagni L et aL. Early detection of skin and muscular involvement in Lafora disease. J Neurol 1991; 238: 217-20.

59. Lauria G, Cornblath DR, Johansson O, McArthur JC, MeLLgREN SI, Nolano M ET aL. EFNS guidelines on the use of skin biopsy in the diagnosis of peripheral neuropathy. Eur J Neurol 2005; 12: 747-58. 